\title{
Predictors of poor glycemic control in adult with type 2 diabetes in South-Eastern Nigeria
}

\author{
Innocent Chidi Anioke ${ }^{1,2}$, Azubike Nnamdi Ezedigboh ${ }^{3}$, Ogechukwu Calista Dozie-Nwakile ${ }^{4}$, \\ Ikechukwu Johnpaul Chukwu',5, Peculiar Ngozi Kalu ${ }^{6}$
}

1. Clinical Chemistry Unit; Department of Medical Laboratory Sciences, Faculty of Health Sciences and Technology, College of Medicine, University of Nigeria Enugu Campus, Nigeria.

2. Public Health- Health Promotion, School of Health and Wellbeing, Faculty of Health and Social Science, Leeds Beckett University, Leeds, United Kingdom.

3. Chemical pathology Unit; Department of Medical Laboratory Services, University of Nigeria Teaching Hospital Ituku/Ozolla Enugu, Nigeria.

4. Medical Laboratory Microbiology Unit; Department of Medical Laboratory Sciences, Faculty of Health Sciences and Technology, College of Medicine, University of Nigeria Enugu Campus, Nigeria.

5. Biomolecular and Biomedical Sciences, Glasgow Caledonian University, United Kingdom.

6. Department of Chemical Pathology, College of Medicine, Nnamdi Azikiwe University, Nnewi, Nigeria.

\section{Emails:}

zubbyro@yahoo.com;ogechukwu.dozie-Nwakile@unn.edu.ng; ikechukwu.chukwu@unn.edu.ng;

kuliartreasure@yahoo.com

\begin{abstract}
Background: The study investigated predictors of poor glycemic control in type 2 diabetes (T2DM).

Methods: Data on demographics, anthropometric and clinical parameters were collected in a cross-section survey from 140 adults with T2DM, using standard tools/instruments. Glycated hemoglobin (A1C) was assessed as a measure of glycemic control.

Results: Majority $(83.3 \%)$ had poor glycemic control status of which about $95 \%$ constitute the elderly. The elderly $(\mathrm{OR}=5.90$, $95 \% \mathrm{Cl}: 1.66-20.96)$ were more likely associated with poor glycemic control than the non-elderly $(\mathrm{p}=0.006)$. Adjustment for significant predictor variables: Age, waist-hip ratio (WHR), Fasting plasma glucose (FPG) and Systolic blood pressure (SBP), although attenuated the odds (OR=5.00, 95\% CI: 1.19-20.96) of poor glycemic control, it still remained significantly $(p=0.028)$ higher in the elderly. Patients outside tight FPG control significantly $(\mathrm{p}=0.001)$ showed poor glycemic cotrol than those within tight FPG $(\mathrm{OR}=17.39,95 \% \mathrm{Cl}$ : 5.83-51.90), even with attenuated $\mathrm{OR}(\mathrm{OR}=10.85,95 \% \mathrm{Cl}: 3.10-37.96)$ and $(\mathrm{OR}=12.08,95 \% \mathrm{Cl}$ : 3.64-40.09) when non- significant and significant predictor variables were accounted for, respectively.

Conclusion: Age, WHR, FPG, and SBP were significantly associated with differences in glycemic control. The elderly and FPG outside tight control showed significantly increased odds of poor glycemic control status.

Keywords: Type 2 Diabetes Mellitus; glycemic control status; A1C; Elderly; Fasting plasma glucose; glycemic control predictors. DOI: https://dx.doi.org/10.4314/ahs.v19i4.3

Cite as: Anioke IC, Ezedigboh AN, Dozie-Nwakile OC, Chukwn IJ, Kalu PN. Predictors of poor glycemic control in adult with type 2 diabetes in South-Eastern Nigeria. Afri Health Sci. 2019;19(4):2819-2828. https:/ / dx.doi.org/10.4314/abs.v19i4.3
\end{abstract}

\section{Corresponding author:}

Innocent Chidi Anioke, Clinical Chemistry Unit; Department of Medical Laboratory Sciences, Faculty of Health Sciences and Technology, College of Medicine, University of Nigeria Enugu Campus, Nigeria.

Tel:+2348066752664

Email: innocent.anioke@unn.edu.ng

\section{Introduction}

The growing incidence of type 2 diabetes mellitus (T2DM) would likely heighten the prevalence of DM globally, with about $11 \%$ adults (approximately 114 million) currently suffering with T2DM. This is projected to increase to about 150 million by $2040^{1}$, with $80 \%$ of the cases occurring among population in resource-poor settings ${ }^{2}$. In Africa, the estimated prevalence of diabetes is $1 \%$ in rural areas, and about $5 \%$ to $7 \%$ in urban sub-Sa-

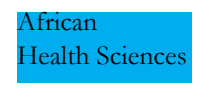

(C) 2019 Anioke et al. Licensee African Health Sciences. This is an Open Access article distributed under the terms of the Creative commons Attribution License (https://creativecommons.org/licenses/BY/4.0), which permits unrestricted use, distribution, and reproduction in any medium, provided the original work is properly cited. 
haran Africa ${ }^{3}$, with Nigeria having the highest burden of the disease ${ }^{4}$. Data from National Survey on Non-Communicable Disease indicates that the prevalence of DM in Nigeria was $2.2 \%$ in $1997^{5}$. In 2015 , this has increased to $5.0 \%{ }^{4}$. Although, the current prevalence of $\mathrm{DM}$ in Nigeria is not well documented, however, this may possibly vary between $8 \%-10 \%{ }^{6}$. Epidemiological evidence indicates an increasing burden of T2DM among adult Nigerian (20-79 years) with about 874000 cases representing $2.0 \%$ estimated prevalence rates in 1990 to 4.7 million cases $(5.7 \%)$ in $2015^{7}$. Females have higher prevalence than males ${ }^{8,9}$. However, prevalence increases with ageing in both sexes ${ }^{8}$.

Regardless of sex, maintaining tight glycemic control among patients is fundamental in preventing complications associated with the disease. Several factors, both clinical and non-clinical factors, have been noted to impact the level of glycemic control in individuals. For instance, there are few non-modifiable (e.g. ethnicity, age, family history/genetic factor, sex) and modifiable (e.g. excess alcohol intake, smoking, physical inactivity, unhealthy diet, educational status, metabolic abnormalities and obesity/overweight) factors reported to influence the outcome of T2DM management ${ }^{4,8}$. Interestingly, prior studies ${ }^{1,10,11}$, have reported ethnic difference particularly in T2DM. Perhaps, given the ethnic disparities in glycemic control ${ }^{12}$ arising from the differences in the distribution of critical risk factors that impact glycemic control such as age ${ }^{1,13}$, sex, social economic status, body mass index $(\mathrm{BMI})^{1}$, lifestyles, processes of care/adherence to treatment ${ }^{14,15,16,18}$, blood pressure and duration of the disease $\mathrm{e}^{16,17,18}$ etc. However, in Nigeria- especially South-Eastern part, there still remains dearth of data regarding glycemic control status, a pivotal step for achieving marked reduction of diabetic complications. Against this background, it became pertinent in this study to identify first, the level of glycemic control status among T2DM patients under treatment. Secondly, investigate the demographic, anthropometric and clinical factor(s) associated with glycemic control in T2DM patients with different diabetic complications in south-east Nigeria, and the extent of association with or without accounting for joint influence of significant predictor variables.

\section{Patients and methods \\ Study design, setting and ethics}

A cross-sectional survey was used for the study. Whilst this design was unable to establish a causal $\operatorname{link}^{19}$ between glycemic control status and studied demographic-clinical variables, it was a useful methodology for comparison and demonstration of the relationship (association) between dependent variable (glycemic control status) and independent (demographics, anthropometrics \& clinical) variables. By this design, it implies that changes in one variable accompany changes in another rather than cause and effect ${ }^{19}$.This study was conducted at the diabetic clinic of Nnamdi Azikiwe University Teaching Hospital, NAUTH, Nnewi. NAUTH is one of the major tertiary hospitals located in South-East of Nigeria. This study received ethical approval in line with the Research Ethics Policy and Procedures of the above named hospital prior to initiation of the study.

\section{Study population and sample size}

The study population consists of clinically confirmed T2DM out-patients under treatment, attending NAUTH for check-up. A sample size of 132 participants was calculated for the study considering absolute precision of $5 \%$ with a design effect of 1.5 using online method as described by Dean et al. ${ }^{20}$. This was based on assumed population size of 1000 for the setting selected, and a documented prevalence of T2DM in South-East Nigeria reported to be $6.7 \%{ }^{21}$. However, a higher number of 150 patients were targeted to account for possible refusals and dropouts during the study.

\section{Sampling procedure}

Out-patients with T2DM who attends the diabetic clinic at NAUTH were recruited based on inclusion and exclusion criteria set for the study. Patients were included if they satisfied the following inclusion criteria: were clinically confirmed T2DM patients within the clinic, are 30 years and above, under treatment for at least one year, and the willingness to participate and is able to give consent. However, those who met these inclusion criteria but were on drugs that could affect glucose metabolism e.g. steroid and B-blockers or pregnant for the females, were also excluded from the study. Informed consent was provided 
by each eligible patient who was willing to participate in the study after the purpose, risk and benefits of the study was explained to all participants. A convenient sampling method was used to select samples of the population between June 2, 2013 and September 28, 2013. Finally, the study comprised data collected from 140 patients who cooperated in every stage of the project.

\section{Data collection}

\section{Demographic and anthropometric assessment}

Patient's clinical characteristics, including duration of T2DM since diagnosis, kind of diabetic complications, and anti-diabetic medications and other concurrent medications received by patients were obtained by review of patients' medical records. The data on patients demographic (age and sex) were also obtained from the record while they underwent a standardised medical examination that included routine anthropometric and clinical assessment. Weight was taken with patients having any heavy wears such as shoes, clothing and belts removed, using digital Soehnle electronic scales (Leifheit AS, Nassau, Germany). Height was measured using a stadiometer with the participant standing erect in bare feet with head in the Frankfurt plane. BMI was calculated by dividing weight (in $\mathrm{kg}$ ) by height squared (in $\mathrm{m}^{2}$ ) and categorized using the WHO classification ${ }^{22}$ into: $<18.5 \mathrm{~kg} / \mathrm{m}^{2}$ (underweight), 18.5-24.9 kg/m2 (normal weight), 25-29.9 $\mathrm{kg} / \mathrm{m} 2$ (overweight), $\geq 30 \mathrm{~kg} / \mathrm{m}^{2}$ (obese). Three BMI categories were created for the purpose of the study by collapsing the under-weight with normal-weight to form a single category. WHR was calculated by dividing waist circumference (WC) by hip circumference (HC) all in centimetre $(\mathrm{cm})$.WC was measured at the level of the iliac crest while the participants breathed out gently using a measuring tape whereas measurement for the $\mathrm{HC}$ was taken at the maximal gluteal protrusion. The blood pressure was measured twice in a sitting position after $5 \mathrm{~min}$ of rest with a mercury Sphygmomanometer according to a standard protocol. The average of the two readings was considered as the blood pressure value in $\mathrm{mmHg}$ for each patient. To ensure reliability and validity of results, all the patients' data were collected using same measuring instrument.

\section{Biochemical laboratory assessment}

About $5 \mathrm{ml}$ of venous blood was taken from each subject in a fasting state (i.e. after overnight fast) by a medical laboratory scientist, who is a member of the research team. About $2 \mathrm{ml}$ of blood sample collected were dis- pensed into EDTA anticoagulant bottle and $3 \mathrm{ml}$ into fluoride oxalate bottle. Whole blood for A1C assay was stored at $4^{0} \mathrm{C}$ up to one week while the FPG was immediately analysed. The quantitative determination of A1C, an index of glycemic control status in this study, was carried out by Direct Enzymatic (fructosyl valine oxidase) method whereas FPG was estimated by glucose oxidase method using Randox kit.

In line with the therapeutic goal of $\mathrm{A} 1 \mathrm{C}$ in diabetes which is to maintain a value $<7 \%$ as recommended by the International Diabetes Federation (IDF) and American College of Endocrinology (ACE) ${ }^{2}$, A1C data was divided into two categories: Good glycemic control $(<7 \%)$

and poor glycemic control ( $\Xi 7 \%$ ). This was to satisfy the purpose of the study.

\section{Data analysis}

All data analyses were performed using SPSS, IBM ${ }^{\circledR}$ SPSS ${ }^{\circledR}$ Statistics for Windows Version 20.0(IBM Corp. Armonk, NY, USA). Descriptive analysis was carried out for the predictor (independent) variables (Age, Sex, BMI, WHR, FPG, Complications, SBP and diastolic blood pressure (DBP)) and outcome (dependent) variable (A1C). The characteristics of the sample were presented as percentage (frequencies) or mean $\pm \mathrm{SD}$ where appropriate, first by demographic, anthropometric and clinical status and then as a function of glycemic control categories. The association between variations in glycemic control status and variations in predictor variables were shown with cross-tabulation, and Pearson Chi-squared, Fisher's exact and student t-tests were used, where appropriate to select significant predictors of good or poor glycemic control status. Binary logistic regression analysis was used to analyze the level of association between significant predictor factors (Age, WHR, FPG and SBP) and the index of glycemic control status (A1C). First, crude odds ratios (ORs) and 95\% confidence intervals (CIs) for each significant predictor variable were calculated using univariable logistic regression. Secondly, the independence of any association was examined by accounting for the influence of non-significant predictor variables such as Sex, BMI, Complications and DBP in a multivariable logistic regression model. Finally, the Odds Ratio and $95 \%$ confidence intervals were calculated to identify predictor(s) of glycemic control status using multivariable logistic regression model including only the significant predictor variables: Age, WHR, FPG, and SBP. $\mathrm{p}<0.05$ was considered statistically significant value for all comparisons. 


\section{Results}

\section{General Characteristics of the studied population}

In Table 1, the descriptive statistics of patient's characteristics were presented. The proportion of males and females in 140 sample studied was made up of approximately equal proportion of patients, with only a difference of $10 \%$. Fewer than half $(41.4 \%)$ of the patients were elderly. Less than $10 \%$ of the sample were underweight/ normal whereas more were either overweight $(55.0 \%)$ or obese (35.7\%) according to WHO classification of obesi- ty. Most of the patients (67.5\%) fall within low WHR. In addition, $61.3 \%$ of the patients presented with different diabetic complications while $38.6 \%$ had no or unknown complications. Greater proportion of the study population $(85.0 \%)$ had FPG outside tight control. The same was true of the index of glycemic control status (A1C). Majority $(115 ; 83.3 \%)$ of the patients showed poor glycemic control status (A1C $\geqq 7 \%)$ while $23(16.7 \%)$ had good glycemic control status (A1C < 7\%).

Table 1: Distribution of demographic and clinical characteristics of the studied patients

\begin{tabular}{|c|c|c|}
\hline Variables & $\mathbf{N}$ & Patients; n (\%) \\
\hline \multicolumn{3}{|l|}{ Demographic } \\
\hline Age (yrs) & 140 & \\
\hline Non elderly ( $<65$ years) & & $82(58.6)$ \\
\hline Elderly ( $=65$ years $)$ & & $58(41.4)$ \\
\hline Sex & 140 & \\
\hline Male & & $77(55.0)$ \\
\hline Female & & $63(45.0)$ \\
\hline \multicolumn{3}{|l|}{ Anthropometric } \\
\hline Body mass Index (BMI) $\left(\mathrm{kg} / \mathrm{m}^{2}\right)^{*}$ & 140 & \\
\hline Underweight/normal $(<25)$ & & $13(9.3)$ \\
\hline Overweight (25-29.9) & & $77(55.0)$ \\
\hline Obese $(\geq 30)$ & & $50(35.7)$ \\
\hline Waist-Hip Ratio(WHR) & 139 & \\
\hline Low & & $66(67.5)$ \\
\hline Medium & & $20(14.4)$ \\
\hline High & & $53(38.1)$ \\
\hline \multicolumn{3}{|l|}{ Clinical } \\
\hline HbA1c (\%) & 138 & \\
\hline Good control $(<7)$ & & $23(16.7)$ \\
\hline Poor control( $(\geq 7)$ & & $115(83.3)$ \\
\hline Fasting plasma glucose $(\mathrm{FPG})(\mathrm{mmol} / \mathrm{l})$ & 140 & \\
\hline Within tight control(3.9-7.2) & & $21(15.0)$ \\
\hline Outside tight control $(<3.9$ or $>7.2)$ & & $119(85.0)$ \\
\hline Complications & 140 & \\
\hline Neuropathy & & $2(1.4)$ \\
\hline Retinopathy & & $38(27.1)$ \\
\hline Nephropathy & & $31(22.1)$ \\
\hline Foot amputation/or ulcer & & $15(10.7)$ \\
\hline Non/unknown & & $54(38.6)$ \\
\hline Blood pressure & 140 & \\
\hline Systolic blood pressure $(\mathrm{mmHg})$ Mean $(\succsim \mathrm{SD})$ & & $144.32( \pm 21.03)$ \\
\hline Diastolic blood pressure $(\mathrm{mmHg})$ Mean $( \pm \mathrm{SD})$ & & $88.19(\longleftarrow 13.15)$ \\
\hline
\end{tabular}

*BMI $=$ weight $(\mathrm{kg}) /($ height $\mathrm{x}$ height $)\left(\mathrm{m}^{2}\right)$, Low-WHR: male $\leq 0.95 \&$ female $\leq 0.80$; medium-WHR: male 0.96 to 1.0 \& female 0.81 to 0.85 ; high-WHR: male $>1.0 \&$ female $>$ 
Potential predictors of differences in glycemic control status

Table 2 shows that among the patients' demographic, anthropometric and clinical variables: age, WHR, FPG, and SBP showed significant association with glycemic control status. Age $(x=7.747, p=0.005)$ showed a significant association with glycemic control. Most $(94.7 \%)$ of the elderly patients ( $\geq 65$ years) tend to show poor glycemic control compared to non-elderly $(75.3 \%)$, indicating that more of the non-elderly $(24.7 \%)$ had good glycemic control than the elderly $(5.3 \%)$. WHR $\left(x^{2}=6.775, \mathrm{p}=\right.$ 0.034) showed a significant association, with most pa- tients with medium or high WHR more likely to show poor glycemic control than those with low WHR who tend to have a good glycemic control status. FPG $x^{2}=$ 36.497, $\mathrm{p}=0.001$ ) showed a significant association with glycemic control. Most of the patients with FPG outside tight control tend to show poor glycemic control while those within tight control were more likely to have a good glycemic control status. Besides, patients associated with increased SBP were significantly more likely to have poor glycemic control, while those with decreased SBP tend to have a good glycemic control (F-ratio $=8.442, \mathrm{p}=$ 0.021). However, other studied variables were not significantly associated with glycaemia control status in T2DM

Table 2: Glycaemic control status with demographic and clinical variables in studied patients

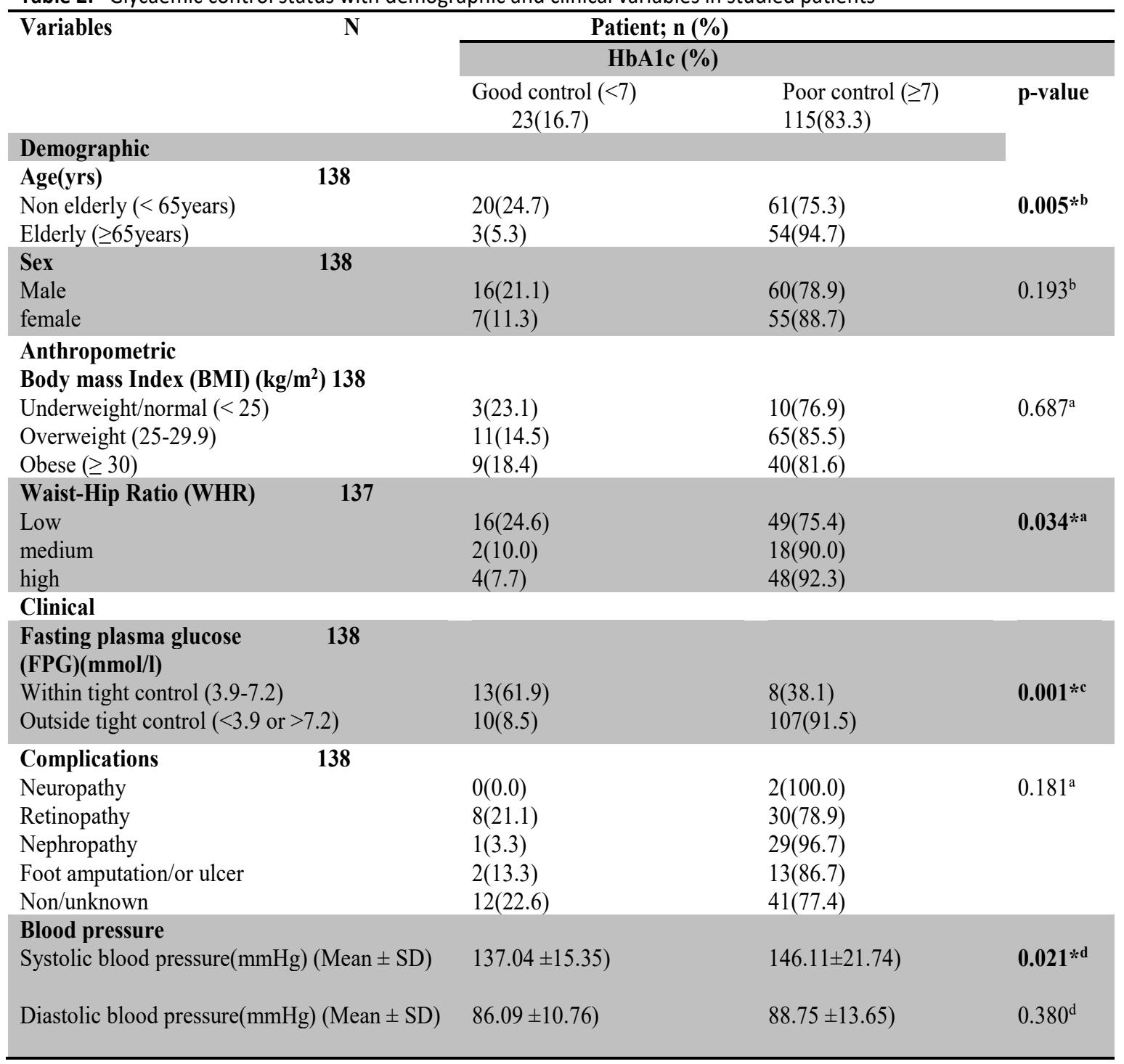

aPearson chi-square test; ${ }^{b}$ Continuity correction; ${ }^{\mathrm{c}}$ Fisher's exact test; ${ }^{\mathrm{d}}$ Students' t-test; ${ }^{*}$ statistically significant value $(\mathrm{p}<0.05)$ 
Level of association of significant predictor variables with glycemic control in T2DM subjects

In Table 3, the analysis using multivariate binary logistic regression model-predicting factors associated with glycemic control in T2DM patients with different complications showed that; age (in model $1 \& 3$ ), WHR (in model 1), and FPG (in model 1, 2 \&3) were significantly associated with glycemic control.

Age: Model 1(table 3), the odds of a patient having poor glycemic control, is 6 times significantly $(p=0.006)$ more likely for elderly than for non-elderly (OR 5.90, 95\% Cl: 1.66-20.96). In model 2 , the significant association between elderly and glycemic control disappeared as the OR for the elderly reduced (OR change from 5.90 to 3.94). This indicate that the elderly patients is not significantly $(p=0.066)$ more likely than non-elderly to report poor glycemic control (OR 3.94, 95\% Cl: 0.91-17.05), when the influence of non-significant predictor variables: sex, BMI, complications and DBP were accounted for. However, in model 3, after adjusting (i.e. accounting) for significant predictor variables (Age, WHR, FPG and SBP jointly associated alone), although it attenuated the odds (OR 5.00, 95\% CI: 1.19-20.96) of poor glycemic control, it still remained significantly $(\mathrm{p}=0.028)$ higher in the elderly compared to the non-elderly (Table 3 ).

WHR: High WHR is significantly $(\mathrm{p}=0.022)$ more likely than low WHR to show poor glycemic control (OR 3.92, $95 \% \mathrm{Cl}: 1.22-12.57)$ in model 1 . However, the overall association between glycemic control status and WHR do not differ significantly either in model 2 or in model 3 (Table 3).

FPG: Across the models (Table 3), the significant for the OR for patients outside tight FPG control has not changed much at all (OR change from 17.39(model 1) to 10.85(model 2) to 12.08 (model 3). They are still significantly more likely to show poor glycemic control than patients with FPG within tight control. This indicates that FPG is an independent predictor factor significantly associated with glycemic control status, and patients outside tight FPG control are more than 10 times more likely to show poor glycaemia control than those within tight FPG control.

Table 3: Multivariate binary logistic regression model-predicting demographic and clinical factors linked with glycemic control status

\begin{tabular}{|c|c|c|c|c|c|c|}
\hline & glycaemic & control s & tatus & & & \\
\hline & Model 1 & p-value & Model 2 & p-value & Model 3 & p-value \\
\hline $\begin{array}{l}\text { Demographic } \\
\text { predictor variable }\end{array}$ & & & & & & \\
\hline $\begin{array}{l}\text { Age (yrs) } \\
\text { Non elderly } \\
\text { Elderly }\end{array}$ & $\begin{array}{l}\operatorname{Ref}(1.00) \\
5.90(1.66-20.96)\end{array}$ & $0.006^{*}$ & $\begin{array}{l}\operatorname{Ref}(1.00) \\
3.94(0.91-17.05)\end{array}$ & 0.066 & $\begin{array}{l}\operatorname{Ref}(1.00) \\
5.00(1.19-20.96)\end{array}$ & $0.028 *$ \\
\hline $\begin{array}{l}\text { Anthropometric } \\
\text { predictor variable }\end{array}$ & & & & & & \\
\hline $\begin{array}{l}\text { WHR } \\
\text { Low } \\
\text { medium } \\
\text { high }\end{array}$ & $\begin{array}{l}\operatorname{Ref}(1.00) \\
2.94(0.61-14.07) \\
3.92(1.22-12.57)\end{array}$ & $\begin{array}{l}0.177 \\
\mathbf{0 . 0 2 2} *\end{array}$ & $\begin{array}{l}\operatorname{Ref}(1.00) \\
2.82(0.38-20.97) \\
2.29(0.38-13.99)\end{array}$ & $\begin{array}{l}0.312 \\
0.368\end{array}$ & $\begin{array}{l}\operatorname{Ref}(1.00) \\
2.65(0.42-16.88) \\
1.82(0.47-6.98)\end{array}$ & $\begin{array}{l}0.302 \\
0.386\end{array}$ \\
\hline $\begin{array}{l}\text { Clinical predictor } \\
\text { variables }\end{array}$ & & & & & & \\
\hline $\begin{array}{l}\begin{array}{l}\text { Fasting plasma } \\
\text { glucose }(\mathbf{F P G}) \\
(\mathbf{m m o l} / \mathbf{l})\end{array} \\
\text { Within tight control } \\
(3.9-7.2) \\
\text { Outside tight control } \\
(<3.9 \text { or }>7.2)\end{array}$ & $\begin{array}{l}\operatorname{Ref}(1.00) \\
17.39(5.83-51.90)\end{array}$ & $0.001 *$ & $\begin{array}{l}\operatorname{Ref}(1.00) \\
10.85(3.10-37.96\end{array}$ & $0.001 *$ & $\begin{array}{l}* \quad \operatorname{Ref}(1.00) \\
12.08(3.64-40.09)\end{array}$ & $0.001 *$ \\
\hline $\begin{array}{l}\text { Blood pressure } \\
\text { Systolic blood } \\
\text { pressure (mmHg) }\end{array}$ & $1.02(0.99-1.05)$ & 0.063 & $1.02(0.98-1.06)$ & 0.331 & $1.01(0.98-1.05)$ & 0.377 \\
\hline
\end{tabular}




\section{Discussion}

Type 2 diabetes mellitus accounts for approximately 80$90 \%$ of all cases of diabetes in adults with varying factors affecting glycemic control level, a key strategy in preventing as well as reducing possible complications arising from the disease. The present study investigated the glycemic control status among T2DM adult patients under treatment, association of demographic, anthropometric and clinical factor(s) with glycemic control in T2DM patients with complications, and the extent of association with or without accounting for joint influence of significant predictor variables.

The study revealed that age, WHR, FPG as well as SBP distribution were significantly associated with differences in glycaemic control- assessed with A1C Measurement. Elderly patients, patients with high WHR and a FPG outside the tight control range $(<3.9$ or $>7.2 \mathrm{mmol} / \mathrm{l})$ have significantly increased odds of poor glycemic control compared to non-elderly, patient with medium/low WHR and FPG within the tight control range of 3.9$7.2 \mathrm{mmol} / 1$, respectively. However, after adjustment for significant predictor variables, only elderly patients and patients with FPG outside the tight control range were still significantly associated with increased odds of poor glycaemia control status. These results, incongruent with other previous studies, indicate that the glycemic control status of diabetic patients is significantly linked with age $^{12,18,23,24}$, and FPG of each patient in question ${ }^{23}$. In the contrary, these results disagree with a prior study ${ }^{25}$, that reported no significant association between age groups and glycemic control.

According to cumulative advantage/disadvantage theory, several explanatory factors relating to health outcomes are subject to modification with ageing ${ }^{26}$. Metabolic health changes associated with advanced age could possibly be a critical factor to consider in light of the suboptimal or poor glycemic control status noted among such a heterogeneous group as the elderly ${ }^{27}$. For instance, normal aging is characterized with a progressive glucose intolerance arising from an impairment linked to glucose-induced insulin release. This age-related impairment has been attributed to substantial decrease in beta-cell response to anti-diabetic agent (e.g. glucose-dependent insulinotropic polypeptide), particularly in hyperglycaemic state ${ }^{28}$. Moreover, elderly patients have been reported to exhibit poor compliance to recommended therapy required to achieve optimal glycemic control ${ }^{18}$. Undoubtedly, however, most treatment options for this group has been derived to a large extent from data generated among the nonelderly ${ }^{29,30,31}$ given the few evidence-based research works from which guidelines for treatment for the elderly patients can be derived ${ }^{27}$. Arguably, such treatment approach in terms of achieving better glycemic control may likely favour the nonelderly patients than their elderly counterparts, which is the case in the present study. This finding is not consistent with one prior study ${ }^{31}$. However, in elderly patients, more complex factors may be influencing the glycemic control status than in the younger adults as our finding suggests.

BMI in the current study was not a significant predictor of poor glycemic control, even though higher proportion of overweight/obese individuals were associated with poor glycemic control status. WHR (an indicator of central adiposity) was found to be a significant potential predictor of poor glycemic control in diabetes. The prediction of T2DM, and perhaps its attendant poor glycemic control, has been suggested by clinical evidence to be better and stronger with indicators of central adiposity (e.g. WHR) than with general adiposity (e.g. BMI) ${ }^{32,33}$. This was attributed to the fact that central adiposity has been linked with impairment in glucose-insulin homeostasis leading to a decrease in both glucose tolerance and insulin-stimulated glucose disposal ${ }^{33}$. According to Vazquez et al. ${ }^{33}$, the ability of both adiposity indicators to predict T2DM may vary by demography such as ethnicity, sex and age. Perhaps, this is why our finding on BMI and poor glycemic control did not echo a prior study ${ }^{24}$, which reported BMI as a significant predictor of poor glycemic control. However, the finding on WHR as a significant predictor of poor glycemic control lends support to the clinical evidence, suggesting that indicators of central adiposity are better predictors of poor glycemic control in type 2 $\mathrm{DM}^{32,33,34}$.

Majority of patients in the study had FPG outside tight control. This, of course, was not contrary to expectation, because previous documented evidence had reported that FPG have good predictive value for overall glycemic control $^{35,36}$. Thus, in the context of existing body of research, the result in congruent with ${ }^{35,36}$ indicates that FPG is one biomarker that has a strong positive association with glycated haemoglobin (A1C). 
Patients associated with increased SBP were significantly linked with poor glycemic control, while those with decreased SBP tend to show good glycemic control. For diabetic patients and other high-risk patients, the recommended guidelines suggested lowering the systolic blood pressure to a treatment goal less than $130 \mathrm{~mm} \mathrm{Hg}^{37}$. The guideline seems to uphold "the lower the better" approach to blood pressure control in diabetic individuals ${ }^{38}$, which the present finding, in agreement with prior study ${ }^{39}$, is in line with. Although, some authors have argued that below this recommended blood pressure guideline $(130 / 80 \mathrm{~mm}$ $\mathrm{Hg}$ ) in diabetes, increased risk for poor outcomes have also been recorded ${ }^{40}$.

\section{Limitation of the study}

We recognised that multiple factors may interact to affect glycemic control. Our model, however, examined just a few of those; demographics (age and sex), anthropometric (BMI and WHR) and clinical (FPG and BP) factors, variables capable of yielding data less subject to bias. Social-economic determinants (confounders) of glycemic control status such as lifestyle/behavioural factors (diet, exercise, smoking etc), educational level, social class status, knowledge of self-care, etc were left unexplained, because information on such patients' data are meant to be collected with a questionnaire, a tool often subject to social desirability bias ${ }^{41}$ and recall bias, especially in study area such as ours. Postprandial plasma glucose (PPG) was not assessed in the study. Although PPG is a better predictor of overall glycemic control than FPG, but that, is in the absence of HbA1c. However, whether FPG or PPG is a better predictor of glycemic control is still a subject of debate ${ }^{42}$. Moreover, given that $\mathrm{HbA1c}$ was the measure of glycemic control used in the present study, we included only FPG, the most common routine test for day-to-day monitoring of diabetic patients in South-Eastern Nigeria hospitals. Besides, FPG is considered to be the major contributor for glycation of haemoglobin and can account for a shift in glycemic status of the patients ${ }^{43}$. However, our result may have been affected by those unaccounted possible confounders. As such, given the few predictor variables considered in our study, we wish to submit that our findings alone may not be sufficient enough to explain the difference in glycemic control in adult with T2DM. Furthermore, no causal associations between potential predictor factors and poor glycemic control can be drawn because of the cross-sectional design adopted in the study. Besides, this study did not account for the differences in drug treatment between various groups, a factor that may partly explain the differences in glycemic control status, particularly among elderly and non-elderly groups. Finally, as a single centre study which adopted convenience sampling technique for recruitment of patients rather than random sampling, generalization of findings should be undertaken with caution-as only the patients who visited the NAUTH diabetic clinic during the period of study were included.

\section{Strength and clinical implication of the study}

This study is first of its kind to show the level of glycemic control status among adult with T2DM in south-east Nigeria, suggesting that older adult ( $\geq 65$ years) show poor glycemic control status regardless of complications and the treatment options. Interestingly, also the study revealed those significant predictors (age, WHR, FPG and SBP) of poor glycemic control among the population. Findings indicate that these potential predictors of poor glycemic control require adequate consideration during the course of clinical management of diabetes in order to improve, especially elderly patients' outcome. Of greater interest is the practical implication of these findings, which apparently underpins the increasing body of literature advocating a tailored management of the disease for elderly patients ${ }^{44,45}$.

\section{Conclusion}

Taken together, age, WHR, FPG and SBP were significantly associated with differences in glycemic control. The study revealed that the level of DM management in south-east Nigeria is unsatisfactory, as majority showed poor glycemic control status, particularly among the elderly DM patients. Therefore, the complex interactions between potential predictors of poor glycemic control among this age group -beyond the ones considered in this study- require further investigation in a wider scale such as population-based study in a low-income setting as ours. This may help to come up with improved treatment interventions and strategies that would favour better glycemic control status, as such reduce the risk of diabetic complication among the elderly population.

\section{Competing interests}

The authors have no competing interests to declare. 


\section{References}

1. International Diabetes Federation. The IDF Diabetes Atlas. ${ }^{\text {th }}$ ed. Brussels: Belgium, international Diabetes Federation, 2017.

2.Negandhi PH, Ghouri N, Colhoun HM, Fischbacher CM, Lindsay RS, et al. Ethnic Differences in Glycemic Control in People with Type 2 Diabetes Mellitus Living in Scotland. PLoS One, 2013, 8(12): e83292.

3. Kengne AP, Amoah AG, Mbanya JC. Cardiovascular complications of diabetes mellitus in sub-Saharan Africa. Circulation 2005; 112: 3592-3601

4. Oputa,R. \& Chinenye, S. Diabetes in Nigeria - a translational medicine approach. African Journal of Diabetes Medicine, 2015; 23(1):7-10.

5 Akinkugbe,O. \& Akinyanju,O. "Final report.National Survey on Non-Communicable Disease," Tech. Rep., Federal Ministry of Health, Lagos, Nigeria.1997.

6. Ogbera AO, Ekpebegh C. Diabetes mellitus in Nigeria: The past, present and future. World J Diabetes 2014; 5(6): 905-911

7. Adeloye, D., Ige, J., Aderemi, A., et al. Estimating the prevalence, hospitalisation and mortality from type 2 diabetes mellitus in Nigeria: a systematic review and meta-analysis. BMJ Open, 2017;7.

8. Ekpenyong,C., Akpan,U., Ibu,J.\& Nyebuk,D. Gender And Age Specific Prevalence And Associated Risk Factors Of Type 2 Diabetes Mellitus In Uyo Metropolis, South Eastern Nigeria. Diabetologia Croatica, 2012; 41(1):17-28.

9. Ogedengbe,S. \& Ezeani,I. Profile of metabolic abnormalities seen in patients with type 2 diabetes mellitus and their first degree relatives with metabolic syndrome seen in Benin City, Edo state Nigeria. Journal of Diabetes \& Metabolic Disorders, 2014; 13:61.

10. Shaw JE, Sicree RA, Zimmet PZ . Global estimates of the prevalence of diabetes for 2010 and 2030. Diabetes Res Clin Pract, 2010; 87: 4-14. PubMed

11. Hall LML, Sattar N, Gill JMR (2008) Risk of metabolic and vascular disease in South Asians: potential mechanisms for increased insulin resistance. Future Lipidology 3: 411-424.

12. Harris MI, Eastman RC, Cowie CC, Flegal KM, Eberhardt MS: Racial and ethnic differences in glycemic control of adults with type 2 diabetes. Diabetes Care 1999, 22:403-408.

13. Mukhopadhyay B, Forouhi NG, Fisher BM, Kesson CM, Sattar N: A comparison of glycaemic and metabol- ic control over time among South Asian and European patients with Type 2 diabetes: results from follow-up in a routine diabetes clinic. Diabet Med, 2006; 23: 94-98.

14. Fischbacher CM, Bhopal R, Steiner M, Morris AD, Chalmers $\mathrm{J}$ : Is there equity of service delivery and intermediate outcomes in South Asians with type 2 diabetes? Analysis of DARTS database and summary of UK publications. J Public Health (Oxf), 2009; 31: 239-249.

15. McElduff P, Edwards R, Burns JA, Young RJ, Heller R, Long B, Jones G, New JP: Comparison of processes and intermediate outcomes between South Asian and European patients with diabetes in Blackburn, North-West England. Diabet Med, 2005;22: 1226-1233.

16. Hartz A, Kent S, James P, Xu Y, Kelly M, Daly J. Factors that influence improvement for patients with poorly controlled type 2 diabetes. Diabetes Res Clin Pract. 2006;74(3):227-32.

17. Mendes Beatriz Valverde Ana, Fittipaldi Antônio Saraiva João, Neves Celestino Silva Raimundo, Chacra Roberto Antônio, Moreira Jr Duarte Edson. Prevalence and correlates of inadequate glycaemic control: results from a nationwide survey in 6,671 adults with diabetes in Brazil. Acta Diabetologica. 2010; 47(2):137-145.

18. Chiu C-J, Wray LA. Factors predicting glycemic control in middle-aged and older adults with type 2 diabetes. Prev Chronic Dis 2010;7(1):A08.

19. Crosby,R., DiClemente, R.\&Salazar,L.Research Methods In Health Promotion. USA: Jossey-Bass, 2006

20. Dean, A., Sullivan, K. \& Soe, M. Epidemiologic Statistics for Health version, http://www.openepi.com/ Menu/OE Menu.htm ; 2015[accessed 11.02.16].

21. Osuji, C. U. , Nzerem, B. A., Dioka, C. E., Meludu, S. C. \& Onwubuya, E. I. "Prevalence of diabetes mellitus in a group of women attending "August meeting" at Naze South East Nigeria," Journal of Diabetes Mellitus, 2012;2(3), 321- 326.

22. WHO. Obesity: preventing and managing the global epidemic. Report of a WHO consultation. World Health Organ Tech Rep Ser, 2000; 894:i-xii:1-253.

23. Emmanuel M. M, Alexey M., Boyd M., Charles M. Glycaemic control in diabetic patients in Zambia, Pan African Medical Journal. 2014; 19:354 PubMed doi:10.11604/ pamj.2014.19.354.5264.

24. Nichols GA, Hillier TA, Javor K, Brown JB: Predictors of glycemic control in insulin-using adults with type 2 diabetes. Diabetes Care 2000, 23:273-277.

25. Shorr RI, Franse LV, Resnick HE, Di Bari M, John- 
son KC, Pahor M: Glycemic control of older adults with type 2 diabetes: Find Findings from the Third National Health and Nutrition Examination Survey, 1988-1994. J Am Geriatr Soc 2000, 48:264-267.

26. Dannefer D. Cumulative advantage/disadvantage and the life course: cross-fertilizing age and social science theory. J Gerontol B Psychol Sci Soc Sci 2003;58(6):S327-37.

27. Bhargava,A., Chan,V., Kimball,ES., \& Oyer,DS. Effects of Age on Glycemic Control in Patients With Type 2 Diabetes Treated with Insulin Detemir: A Post-Hoc Analysis of the PREDICTIVETM 303 Study. Drugs Aging, 2016; 33:135-141. PubMed

28. Meneilly,GS., Ryan,AS., Minaker,KL., \& Elahi,D. The Effect of Age and Glycemic Level on the Response of the b-Cell to Glucose-Dependent Insulinotropic Polypeptide and Peripheral Tissue Sensitivity to Endogenously Released Insulin. Journal of Clinical Endocrinology and Metabolism 1998;83(8): 2925- 2932

29. Sinclair A, Morley JE. How to manage diabetes mellitus in older persons in the 21 st century: applying these principles to long term diabetes care. J Am Med Dir Assoc. 2013;14(11):777-80.

30. Kirkman MS, Briscoe VJ, Clark N, et al. Diabetes in older adults: a consensus report. Diabetes Care. 2012;35(12):2650-64.

31. J. Barrot-de la Puente, M. Mata-Cases, J. Franch-Nadal,X. Mundet-Tudur, A. Casellas, J. M. Fernandez-Real, $\&$ D. Mauricio. Older type 2 diabetic patients are more likely to achieve glycaemic and cardiovascular risk factors targets than younger patients: analysis of a primary care database. Int J Clin Pract, 2015; (69): 12, 1486-1495

32. Sushil,MI., Muneshwar,JN.\&Khan,ST. To Study Body Mass Index, Waist Circumference, Waist Hip Ratio,Body Adiposity Index And Lipid Profile Level In Patients With Type-2 Diabetes Mellitus. OSR Journal of Dental and Medical Sciences 2015;14(5): 98-101

33. Vazquez, G., Duval,S., Jacobs,DR., \& Silventoinen,K. Comparison of Body Mass Index, Waist Circumference, and Waist/Hip Ratio in Predicting Incident Diabetes: A Meta-Analysis. Epidemiologic Reviews, 2007;9: 115-128

34. Ashok,K . Prevalence of Glycemic Status, Obesity \& Waist Circumference in Punjabi Type 2 Diabetics. Journal of Exercise Science and Physiotherapy, 2013; 9(1): 1-5.

35. Ketema,EB. \& Kibret,KT. Correlation of fasting and postprandial plasma glucose with $\mathrm{HbA} 1 \mathrm{c}$ in assessing glycemic control; systematic review and meta-analysis. Archives of Public Health;2015; 73:43 PubMed DOI 10.1186/ s13690-015-0088-6

36. Valensi, P., Husemoen,LN., Weatherall,J.\& Monnier,L. Association of postprandial and fasting plasma glucose with HbA1c across the spectrum of glycaemic impairment in type 2 diabetes. Int J Clin Pract. 2017;71:e13041. 37. Standards of medical care in diabetes-2010. Diabetes Care 2010;33(suppl 1):S11-61.

38. Rutter MK, Nesto RW. Blood pressure, lipids and glucose in type 2 diabetes: how low should we go? Re-discovering personalized care. Eur Heart J, 2011;32:2247-55. 39. Yoda, K., Inaba,M., Hamamoto,K., Yoda,M., Tsuda,A., Mori,K., Yamada,S., Emoto,M., Koyama,H.\& Imanishi,Y. Association between glycemic control and morning blood pressure surge with vascular endothelial dysfunction in type 2 DM patients. Diabetes Care, 2013 (Publish Ahead of Print, published online December 2, 2013)

40. Vamos,EP., Matthew Harris,M., Millett,C., Pape,UJ., Khunti,K., Vasa Curcin,V., Molokhia,M.\& Majeed,A. Association of systolic and diastolic blood pressure and all cause mortality in people with newly diagnosed type 2 diabetes: retrospective cohort study. BMJ, 2012; 345:e5567. 41. Adams SA, Matthews CE, Ebbeling CB, Moore CG, Cunningham JE, Fulton J, et al. The effect of social desirability and social approval on self-reports of physical activity. Am J Epidemiol 2005;161(4):389-98. PubMed

42. Ketema,EB. \& Kibret, KT. Correlation of fasting and postprandial plasma glucose with $\mathrm{HbA1c}$ in assessing glycemic control; systematic review and meta-analysis. Archives of Public Health, 2015; 73:43

43. Patel,S.,Nanda,R., Sahoo,S. \& Mohapatra,E. Predictive Role of Fasting and PostPrandial Glucose towards Glycemic Control. National Journal of Laboratory Medicine, 2016; 5(4): BO15-BO21

44. Hainer TA. Managing older adults with diabetes. $J A m$ Acad Nurse Pract 2006;18(7):309-317.

45. Brown AF, Mangione CM, Saliba D, Sarkisian CA; California Healthcare Foundation/American Geriatrics Society Panel on Improving Care for Elders With Diabetes. Guidelines for improving the care of the older person with diabetes mellitus. J Am Geriatr Soc 2003;51(5 Suppl Guidelines):S265-80. 